\title{
Characterisation of Novel Topical Olive Oil Oleohydrogel Hybrid for Controlled Drug Release
}

\author{
Dipanjana Ash*1, Sutapa Biswas Majee², Dhruti Avlani ${ }^{3}$ \\ 1. Department of Pharmaceutical Technology, Brainware University, 398, Ramkrishnapur Road, Barasat, Kolkata, West Bengal, India. \\ 2. Department of Pharmacy, NSHM Knowledge Campus, Kolkata-Group of Institutions, 124 B.L.Saha Road, Kolkata, West Bengal, India. \\ 3. KLE College of Pharmacy, Rajaji Nagar, Bengaluru, Karnataka, India. \\ *Corresponding author's E-mail: dipanjanaash16@gmail.com
}

Received: 11-06-2020; Revised: 18-08-2020; Accepted: 29-08-2020.

DOI: $10.47583 /$ ijpsrr.2020.v64i01.024

\begin{abstract}
Oleohydrogel hybrid, a novel combination of lipophilic oleogel and hydrophilic hydrogel phases is assumed to achieve the benefits of both the gel phases and exhibit pseudo plastic flow behavior with an excellent drug release profile thereby providing an unique topical base. The present study deals with the development and characterization of olive oil based paracetamol-loaded Span 40Tween 80-HPMCK15M oleohydrogel hybrid. Olive oil based Span 40-Tween 80 oleohydrogel hybrids were prepared by combining the principles of solid fiber mechanism and hot gelation technique and subjected to rheological study, gelation behavior, thermal stability and drug release profile. FT-IR and hemocompatibility studies revealed compatibility among the gel hybrid components. Gelation time of oleohydrogel hybrids decreased with increase in HPMCK15M concentration. They exhibited pseudo-plastic flow with satisfactory thermal stability. The olive gel hybrids with predominantly matrix structure demonstrated improved drug release profile via non-Fickian diffusion compared to their native Span-Tween based oleogels. The best formulation (OHO*1) however followed concentration-independent zero order kinetics with desirable t50 value of $3.6 \pm 0.2 \mathrm{hrs}$. Oleohydrogel hybrid shows promise as a controlled release topical base with good rheological behavior, thermal stability and drug release profile.
\end{abstract}

Keywords: HPMCK15M, gel-matrix, non-Fickian diffusion, oleohydrogel hybrid, pseudo plastic flow, topical base.

\section{INTRODUCTION}

P hysical, chemical, bio-chemical, immunological factors along with barrier properties of the stratum corneum constitute major obstacles to drug penetration across skin. ${ }^{1}$ An ideal topical drug delivery vehicle should facilitate drug transport through the skin by virtue of its ability to alter the barrier properties through interaction. ${ }^{2}$ Therefore, fabrication of suitable drug delivery vehicle or base possess a great challenge to the pharmaceutical formulator and scientist. A welldeveloped novel semi-solid topical base, oleohydrogel hybrid or bigel, comprising of lipophilic oleogel and hydrophilic hydrogel phases will ideally possess benefits of both the gel phases and may promote enhanced hydration, natural fluidization of the skin and simultaneous transport across the stratum corneum barrier. The unhindered diffusion of hydrophilic component, lipophilic nature of oil and penetration enhancing property of non-ionic surfactant help in achieving beneficial therapeutic effect on skin. Other advantages of this novel topical base include ability to incorporate both hydrophilic and lipophilic drugs, cooling effect, moisturizing effect, easy spreadability, emolliency, water wash ability upon application to the skin and microbiological stability. ${ }^{3-4}$

Several studies have reported bigel formation with natural and synthetic hydrophilic polymers like guar gum, Carbopol, HPMC, PVPK30 and organogelators like Span 60, Span 40 and Span 80..$^{5-8}$
No previous attempts have been made to utilise Span 40 in combination with Tween 80 and HPMC K15M in the development and characterisation of olive oil based oleohydrogel hybrids for topical drug delivery. Topical olive oil preparations are known to exert beneficial effects on skin due to presence of various beneficial components and antioxidants. Moreover, olive oil is compatible with skin $\mathrm{pH}$ and no adverse effects have been reported so far. ${ }^{9-10}$ Cosmetic creams of $\mathrm{O} / \mathrm{W}$ emulsion type developed with virgin olive oil demonstrated efficiency, safety and cost-effectiveness over other topical preparations. ${ }^{10}$ Olive oil based nanoemulsion of inulin demonstrated better transport than micellar dispersions and aqueous solutions and reportedly possessed hydrophilic-lipophilic balance identical to sebum of hair follciles. ${ }^{11}$

The objective of the present study was to formulate oleohydrogel hybrids from oleogels of olive oil containing Span 40/Tween 80 and HPMCK15M hydrogels by varying proportion of the organogelator (Span 40), surfactant/cogelator (Tween 80) and hydrophilic gelling agent (HPMCK15M) and compare the developed formulations with native oleogels on the basis of rheological and thermal properties, drug release profile and drug release kinetics. An attempt was made to predict the possible changes in the microarchitecture of the novel topical base due to hybrid formation. 


\section{MATERIALS AND METHODS}

\section{Materials}

Olive oil (Park Daniel, India) was purchased from local market. Span 40, Tween 80 and HPMCK15M (gift sample)were of AR grade and obtained from Loba Chemie, Merck Specialty Pvt. Ltd. and Colorcon Asia Pvt. Ltd. respectively. Paracetamol I.P. (PCM) was purchased from Balaji Drugs, Kolkata, India. Double distilled water was used throughout the study, wherever required. For hemocompatibility study, fresh goat blood was collected in heparin coated tubes and stored at $-4 \circ \mathrm{O} C$.

\section{Preparation of oleohydrogel hybrid ${ }^{5}$}

Oleogels were prepared by dissolving accurately weighed paracetamol (2\%w/v for drug-loaded batches), Tween 80 and Span 40 (in the ratios of 1:1, 1:2 and 2:1) with continuous stirring at 1000 r.p.m for $1 \mathrm{~h}$ in olive oil maintained at $70^{\circ} \mathrm{C}$. A clear, homogenous solution was obtained which was allowed to cool down subsequently to 25 으 to form oleogels by solid-fiber mechanism having different concentrations $(\% \mathrm{w} / \mathrm{v})$ of organogelator-cogelator. The minimum concentration $(\% \mathrm{w} / \mathrm{v})$ of organogelator-co-gelator required to induce gelation is referred to as critical gelator concentration (CGC) for olive oil. Hydrogels were prepared with three different concentrations of HPMCK15M $(1.5,3.0$ and $4.5 \% \mathrm{w} / \mathrm{v})$ by hot gelation method. The oleogel demonstrating best performance was selected for the preparation of oleohydrogel hybrid by mixing oleogel (in the sol state) with hydrogel at $60 \circ \mathrm{C}$ (in the ratio of $1: 1$ ) with continuous stirring at 500 r.p.m. The formulations were stored in glass vials at $25^{\circ} \mathrm{C}$. The composition of oleogels (OG and $\left.\mathrm{OG}^{*}\right)$, hydrogels and oleohydrogel hybrids (OHO and $\mathrm{OHO}^{*}$ ) is given in Table 1.

Table 1: Composition (\%w/v) of olive oil based formulations

\begin{tabular}{|c|c|c|c|c|c|c|}
\hline Batch & PCM & Span 40 & Tween 80 & Olive oil & HРMCK15M & Water \\
\hline OG* 1 & 2 & 5 & 5 & 88 & - & - \\
\hline OG*2 & 2 & 6.67 & 3.33 & 88 & - & - \\
\hline OG*3 & 2 & 3.33 & 6.67 & 88 & - & - \\
\hline OG* 4 & 2 & 6 & 6 & 86 & - & - \\
\hline OG* 5 & 2 & 8 & 4 & 86 & - & - \\
\hline OG* 6 & 2 & 4 & 8 & 86 & - & - \\
\hline OG* 7 & 2 & 7 & 7 & 84 & - & - \\
\hline OG* 8 & 2 & 9.33 & 4.67 & 84 & - & - \\
\hline OG* 9 & 2 & 4.67 & 9.33 & 84 & - & - \\
\hline $\mathrm{OHO} * 1$ & 2 & 8 & 4 & 86 & 1.5 & 98.5 \\
\hline $\mathrm{OHO} * 2$ & 2 & 8 & 4 & 86 & 3 & 97 \\
\hline $\mathrm{OHO} * 3$ & 2 & 8 & 4 & 86 & 4.5 & 95.5 \\
\hline
\end{tabular}

OGs and OHOs are the corresponding blank gels of drug-loaded oleogels and oleohydrogel hybrids respectively.

\section{Compatibility study of gel formulations}

\section{Fourier- transformed infrared (FT-IR) spectroscopy}

Infrared spectroscopy of oleohydrogel components and trial batches of the formulations (OG* 5 and $\mathrm{OHO}^{*} 1$ ) were carried out by FT-IR spectroscopy (Bruker, Alpha -T, Germany) in attenuated total reflectance mode in the range of $4000-500 \mathrm{~cm}^{-1}$.

\section{Hemo compatibility study}

Accurately weighed 1gm of oleogel / oleohydrogel hybrid was placed inside dialysis tubing ( Medical Extrusion Technologies), immersed in $50 \mathrm{ml}$ of normal saline and incubated at $37{ }^{\circ} \mathrm{C}$ for $1 \mathrm{~h}$ in a shaker incubator(Orbital Shaking Incubator Table Top, PLT-144, Tanco ${ }^{\circ} \mathrm{P} L$ Tandon $\& \mathrm{Co})$ so as to allow the leaching of components from the gels. A small volume of leachant $(0.5 \mathrm{ml})$ was then diluted with $0.5 \mathrm{ml}$ of diluted goat blood (prepared by diluting $8 \mathrm{ml}$ of fresh goat blood with $10 \mathrm{ml}$ of normal saline) followed by the addition of $9 \mathrm{ml}$ of normal saline. The mixture was then incubated at $37^{\circ} \mathrm{C}$ for $1 \mathrm{~h}$ followed by centrifugation at 3000 r.p.m for 10 mins. Positive and negative controls were prepared by replacing leachant with $0.1 \mathrm{~N} \mathrm{HCl}$ and normal saline respectively. The supernatant was analysed spectrophotometrically at $545 \mathrm{~nm}(1800$ UV visspectrometer, Shimadzu Scientific Instruments Inc., India). The test measures \% hemolysis in the presence of formulation. The percent of hemolysis is calculated by Eq. 1.

$$
\begin{gathered}
\% \text { Hemolysis }=(\text { OD test }- \text { OD negative }) \div \\
(\text { OD positive }- \text { OD negative }) \text {.........eq. } 1
\end{gathered}
$$

OD test= optical density of test sample, OD positive= optical density of positive control, OD negative= optical density of negative control.

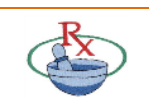




\section{Characterization of gel formulations}

\section{Physico-chemical characterisations}

\section{Organoleptic evaluation}

The freshly prepared gel formulations were subjected to organoleptic evaluation on the basis of color, odor, opacity and appearance.

\section{Evaluation of applicability parameters ${ }^{13-14}$}

Applicability parameters for topical preparation include determination of extrudibility and spreadability. Extrudibility of native oleogels and oleohydrogel hybrids was studied by filling the formulation into a collapsible tube followed by measuring the distance travelled by the ribbon of extruded gel in 10s (Eq. 2). Spreadability study was carried out by placing $1 \mathrm{gm}$ of gel formulations between two glass slides of equal thickness, area and diameter $(75 \mathrm{~mm} \times 25 \mathrm{~mm} \times 1 \mathrm{~mm})$. Initial spreading diameter $\left(D_{i}\right)$ was noted. Thereafter, a load of known weight of $10 \mathrm{gm}, 20 \mathrm{gm}, 50 \mathrm{gm}$ or $100 \mathrm{gm}$ was applied individually on the upper slide for $1 \mathrm{~min}$ and the final spreading diameter $\left(D_{f}\right)$ of gels was noted in each case (Eq. 3). Extrudibility and spreadability are expressed as $\mathrm{cm} / \mathrm{s}$ and percentage respectively.

$$
\begin{aligned}
& \text { Extrudibility }=\text { distance travelled by ribbon of gel } \div \\
& 10 \text { s........................eq. } 2 \\
& \% \text { Spreadability }=[(\mathrm{Df}-\mathrm{Di}) \div \mathrm{Di}] \times 100 \\
& \text { eq. } 3
\end{aligned}
$$

\section{pH measurement}

The $\mathrm{pH}$ (at $25^{\circ} \mathrm{C}$ ) was measured by immersing the glass electrode of the digital $\mathrm{pH}$ meter (Mettler Toledo AG) in the prepared formulations.

\section{Drug content study ${ }^{15-16}$}

Drug content was carried out spectrophotometrically at a wavelength of $249 \mathrm{~nm}$ for estimation of percent drug content from calibration curve of PCM in the buffer.

\section{Rheological study ${ }^{8}$}

Rheological behavior of oleogels and oleohydrogel hybrids was studied with Brookfield viscometer (model LVDLI+) at 25으 by varying the shear rate from 1 to 6 r.p.m (spindle no 1) for $1 \mathrm{~min}$ at each r.p.m. Ostwald deWale Power model (Eq. 4) was employed for viscosity modeling for determination of flow behavior index (n) and flow consistency index (k) from the relationship between shear stress $(\tau)$ and shear rate $(\gamma)$.

$$
\mathrm{T}=\mathrm{k} \times \mathrm{\gamma}^{\mathrm{n}} \text {. }
$$
eq. 4

\section{Thermal analysis}

Thermal analysis of oleohydrogel hybrid could not be carried out by any of the above methods except by Differential Scanning Calorimetry probably due to nonuniform flow of molten fraction of oleogel through the hybrid making precise estimation of end point difficult.

\section{Gelation kinetics and kinetics modelling study ${ }^{17}$}

Oleogel in sol state was transferred to Nessler cylinder and the light was allowed to pass through the sample where suspended particles scattered light. Transformation of sol to gel was characterized by increase in turbidity which continued for certain period of time after which there was no further increase in turbidity. The time at which the turbidity attained a constant value is defined as gelation time. The intensity of turbidity was noted at $20 \mathrm{~s}$ interval in Nepheloturbidometer (ELICO ${ }^{\circ}$ CL52D) and expressed in terms of Nepheloturbidity unit (NTU). Gompertz model was employed for modeling of gelation kinetics of gel formulations. This non-linear model indicates a relationship between turbidity intensity (NTU) (Y), concentration of gelator or gelator-surfactant in $\% \mathrm{w} / \mathrm{v}(\rho)$ and time for gelation in $\mathrm{h}(\mathrm{x}) . \alpha$ is defined as non-polar solvent (oil) parameter and is related to gel flexibility whereas $\beta$ indicates organogelator parameter and can be related to gel's thermal stability.

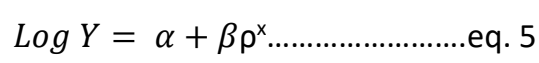

In the above equation, $\rho^{x}$ is defined as

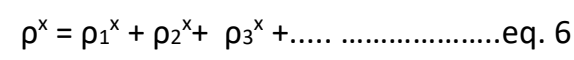

Where $\rho_{1}$ indicates organogelator concentration, $\rho_{2}$ indicates surfactant concentration; $\rho_{3}$ indicates other additive concentration and so on.

\section{Determination of gel-sol transition temperature ${ }^{18}$}

Gel-sol transition temperature $\left(\mathrm{T}_{\mathrm{g}}\right)$ of oleogel was determined by drop ball method. A stainless steel ball having the diameter of $1 / 8$ th inch and a weight of $230 \mathrm{gm}$ was placed over the formulation in a beaker and attached with a melting point apparatus (El-931, Environmental \& Scientific Instruments Co., India). The formulation was heated at a rate of $10 \mathrm{C} / \mathrm{min}$ and the temperature at which the ball started to move through the gel was considered as gel-sol transition temperature $\left(T_{g}\right)$.

\section{Melt flow index ${ }^{19}$}

Melt flow index (MFI) is defined as the ease of melt flow of thermoplastic material in grams over a period of 10 min at a certain standard temperature when checked in melt flow tester (Globe Enterprises, Ghaziabad, India). A fixed weight of oleogel (10 g) was poured into the cylinder and temperature was set at $65^{\circ} \mathrm{C}$ to prevent thermal degradation of gel at higher temperature as specified in standard protocol. Pressure was applied with the piston bar to the cylinder using $10 \mathrm{~g}$ of weight above the piston bar after setting the predefined temperature. Sample flow occurred through the die face in the form of wire and was collected after $10 \mathrm{~min}$ and was weighed to determine its mass.

\section{Differential Scanning Calorimetry (DSC) ${ }^{18}$}

Thermal properties of the gels (including the hybrids) were further examined by using a differential scanning 
calorimeter (Perkin Elmer Pyris Diamond TG/DTA, Singapore) over a temperature range of $32-97^{\circ} \mathrm{C}$ at a heating rate of $0.2 \circ \mathrm{C} / \mathrm{min}$ under an inert $\mathrm{N}_{2}$ gas atmosphere in aluminium crucibles with pierced aluminium lid. The melting parameters [ $T$ onset (onset temperature), $\mathrm{Tm}$ (melting temperature) and $\Delta \mathrm{Hm}$ (melting enthalpy)] were determined from DSC study.

\section{Drug diffusion study}

In vitro drug diffusion study and modelling of release kinetics

Modified Franz diffusion cell was used to perform the in vitro drug release study of prepared oleogels and hybrid through dialysis membrane-60 (HIMEDIA LA 330-5MT, India). Accurately weighed drug-loaded oleogel or its hybrid containing PCM equivalent to $4 \mathrm{mg}$ was placed on the membrane and wetted slightly with phosphate buffer (pH5.8). The buffer solution in the receptor compartment was maintained at $32 \pm 0.5 \circ \mathrm{C}$. An aliquot of $1 \mathrm{~mL}$ was withdrawn every hour for $7 \mathrm{~h}$ and replenished with fresh buffer. Following appropriate dilution, aliquot was analysed spectrophotometrically at $249 \mathrm{~nm}$. The study was performed in triplicate. From drug release profile the time taken for diffusion of $50 \%$ drug i.e., $t_{50}$ value was calculated for all gel formulations for model-independent comparison. Simultaneously, the drug release data of gels were subjected to kinetic modelling to characterise and interpret the drug release pattern and the diffusion mechanism.

\section{Steady-state flux, permeability co-efficient determination ${ }^{20}$}

The measurement of flux across human skin provides a valuable insight into the formulation development of any dermatological product. The steady-state flux of PCM from both oleogels and oleohydrogel hybrid across the artificial dialysis membrane is defined as follows.

$$
\text { SSflux }=\mathrm{dQ} \div \mathrm{dt} \times 1 \div \mathrm{A} \ldots \ldots \ldots \ldots \ldots \ldots \ldots \ldots \text {............... } 7
$$

Where,

SSflux= steady-state flux of drug $\left(\mathrm{mg} / \mathrm{cm}^{2} \cdot \mathrm{hr}\right) ; \mathrm{dQ} / \mathrm{dt}=$ slope of the linear portion of the curve i.e. cumulative amount per unit time $(\mathrm{mg} / \mathrm{hr}) ; \mathrm{A}=$ diffusional area $\left(\mathrm{cm}^{2}\right)$.

Permeability co-efficient is quantified by the following equation

$$
\text { Kp }=\text { SSflux } \div \text { Capp }
$$
.eq. 8

Where,

Capp = initial concentration of the drug in the gel formulation.

In the present study, it was expressed as \%w/v i.e. weight of drug actually present in the volume of gels taken for permeation study.

\section{Accelerated stability study ${ }^{15}$}

Accelerated stability study includes thermo-cycling or freeze/thaw cycling, and syneresis measurements. Thermo-cycling or freeze-thaw cycling method involves incubation of the freshly prepared gel samples at $65 \circ \mathrm{C}$ for 15 mins till the formation of sol state followed by gelation when time was noted. Then these gel formulations were incubated at 4 으 for $15 \mathrm{~min}$ after which stored at $25 \circ \mathrm{C}$ for $48 \mathrm{~h}$. The process was repeated for 6 cycles for oleogel and its hybrids and at the end of each cycle, gelation time was noted.

\section{RESULTS AND DISCUSSION}

\section{Oleohydrogel hybrid formation}

The CGC of Span 40-Tween 80 for olive oil based oleogel was found to be $8 \% \mathrm{w} / \mathrm{v}$ Span- $4 \% \mathrm{w} / \mathrm{v}$ Tween as observed in OG*5. No gelation was induced with gelator/co-gelator in the ratios of $1: 1$ and $1: 2$. No visible signs of phase separation could be found in oleohydrogel hybrids. The dissolution of Span 40 and Tween 80 in the olive oil at $70 \circ \mathrm{C}$ resulted in the formation of clear homogeneous solution but change in the solubility parameter of gelator/co-gelator molecules with lowering of temperature to $25 \circ \mathrm{C}$ resulted in decreased affinities between oil and gelator/co-gelator causing self-assembly of Span/ Tween molecules into aggregates. Tween 80 is reported to improve the solubility of Span in olive oils via the formation of mixed inverse micelles. ${ }^{21}$ Therefore, a three-dimensional network structure of mixed inverse micelles might have been formed by conglomeration of several such aggregates by capturing the oil molecules within solid fibres of organogelator/ co-gelators in oleogels. The critical gelator concentration (CGC) of Span/Tween was found to be $12 \% \mathrm{w} / \mathrm{v}$ (in the ratio $2: 1$ ) (OG* 5) for olive oil. Shah et al. reported CGC of Span 60 and Span 40 to be $18 \% \mathrm{w} / \mathrm{w}$ and $19 \% \mathrm{w} / \mathrm{w}$ respectively for olive oil. ${ }^{19}$ Lower CGC in the present study might be attributed to the solubilising property of Tween 80 as surfactant cogelator. Drug loaded olive oleohydrogel hybrid was prepared by mixing oleogel (OG*5) with hydrogels employing varying concentration of HPMCK15M. HPMCK15M forms a polymer gel network with water with increase in temperature. ${ }^{22}$ Probably, presence of gelator/surfactant-co-gelator promoted formation of uniform, homogeneous creamy white/milky white opaque oleohydrogel hybrid where no phase separation could be detected between organic and aqueous phases. Singh et al. reported that sesame oil based Span 60/ Carbopol bigels were milky white in color and the consistency and smoothness increased with the increase in the organogel concentration. ${ }^{8}$

\section{Compatibility study of gel formulations}

\section{Fourier- transformed infrared (FT-IR) spectroscopy}

FT-IR study was performed for Span 40, Tween 80, PCM, HPMCK15M, olive oil and drug-loaded oleohydrogel hybrid (OHO* 1) (Figure 1). Olive oil showed peaks at 
$2923,2854,1744,1458,1158$ and $720 \mathrm{~cm}^{-1}$. Most of the characteristics peaks were present in the gel formulations, OG* 5 and OHO* 1 . FT-IR analysis (Figure 1 ) revealed an absorption band at $\sim 1744 \mathrm{~cm}^{-1}$, attributed to the presence of a $\mathrm{C}=\mathrm{O}$ stretching vibration due to the ester group of the triglycerides present in the olive oil. The absorption bands at 2916, 2918 and $\sim 2855 \mathrm{~cm}^{-1}$ were due to the $\mathrm{C}-\mathrm{H}$ asymmetric and symmetric stretching vibrations of the Span 40, Tween 80 and olive oil respectively. FT-IR spectra of Span 40 and Tween 80 revealed a broad peak at $3300 \mathrm{~cm}^{-1}$ indicating the presence of $\mathrm{O}-\mathrm{H}$ stretching vibrations. Peaks around
$3200-3600 \mathrm{~cm}^{-1}$ are due to hydrogen bonded $-\mathrm{OH}$ groups of HPMCK15M. Absence of the broad peak at $3300 \mathrm{~cm}^{-1}$ in oleogels and oleohydrogel hybrids may be attributed to intermolecular hydrogen bonding amongst the fatty acyl groups of gelator-co-gelator and oil molecules, which is responsible for imparting strength to the gels. Similar observation was reported by Shah et al. in his work on olive oil based Span 40 and Span 60 organogels. ${ }^{13}$ FTIR analysis of olive oil based oleogels and oleohydrogel hybrids thus indicate compatibility between the oil, organogelator, surfactant and drug.

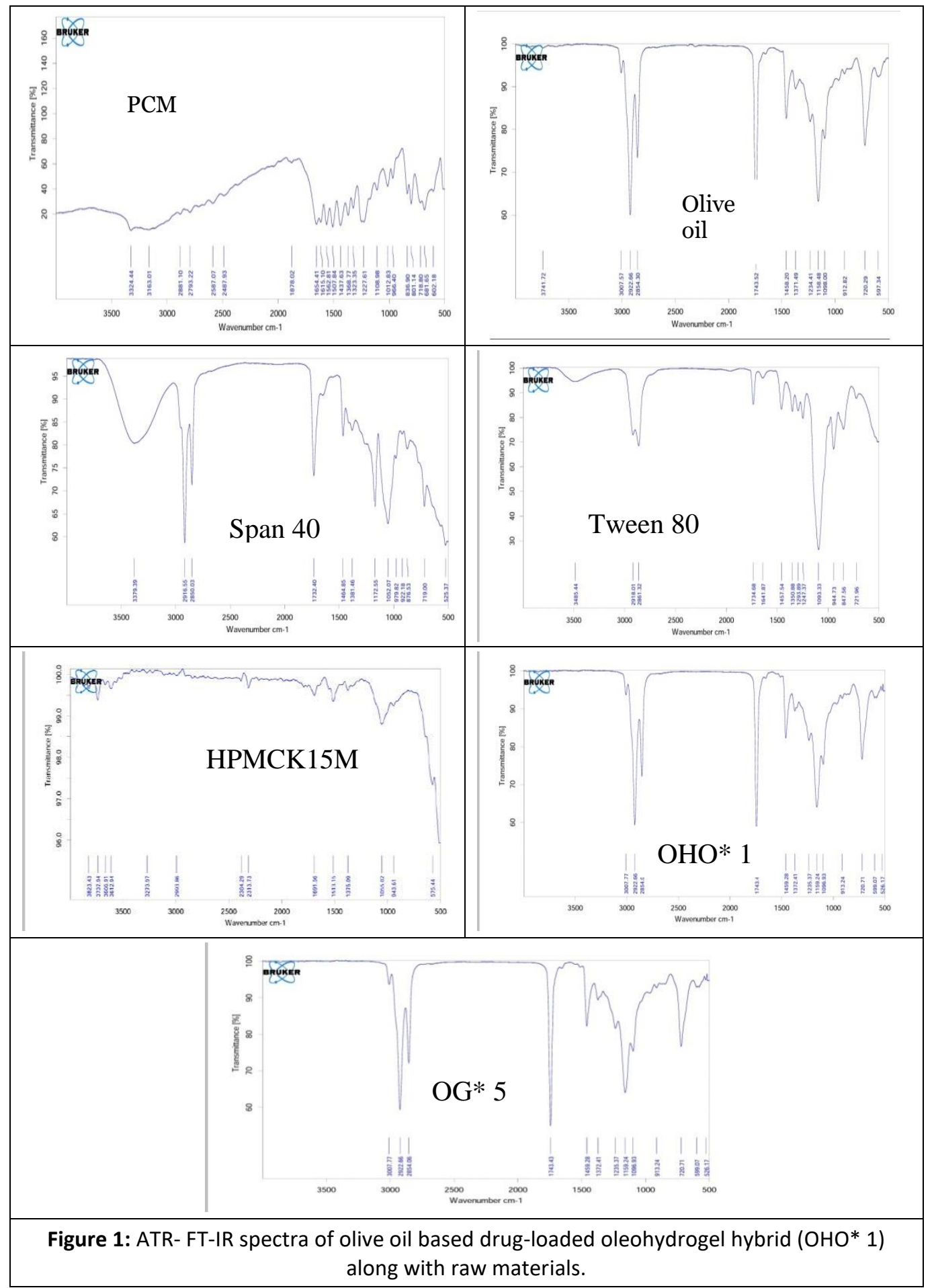




\section{Hemocompatibility study:}

All the prepared formulations were found to be hemocompatible ( $<5 \%$ hemolysis).

\section{Characterization of formulations}

\section{Physico-chemical characterizations}

Span 40/ Tween 80 based olive oleogels were found to be transparent, yellowish-white in color, odorless and smooth-oily in touch. But olive oleohydrogel hybrid acquired opaque milky-white color. The \% spreadability and extrudibility of olive oleohydrogel hybrids were superior to olive oleogels. The $\%$ spreadability of oleogels decreased with increase in concentration in gelator/cogelator. Satapathy et al. reported that the \% spreadability decreased significantly with higher concentration of gelator due to increase in physical strength of the organogels. ${ }^{14}$ From the above studies, it can be proposed that the oleohydrogel hybrids of olive oil may be developed as drug delivery vehicles for topical application. All formulations were found to be compatible with skin $\mathrm{pH}(5.5 \pm 0.9)$ at $25^{\circ} \mathrm{C}$. Drug content for the gel formulations was found to be in the range of $91-94 \%$ w/w.

\section{Rheological study}

Higher the gelator/co-gelator concentration, greater was the viscosity of the gel formulations (Figure 2). The order of viscosity of olive oil based blank formulation is as follows: Oleohydrogel hybrids>oleogels. The order of viscosity of olive oleohydrogel hybrids is as follows: $\mathrm{OHO} 3>\mathrm{OHO} 2>\mathrm{OHO}$. From rheological study it was found that viscosity of olive oil based Span 40/Tween 80 oleogels and oleohydrogel hybrids decreased with increase in shear rate (Figure 2) indicating shear-thinning behavior. Singh et al also reported shear-thinning behavior of sesame oil based metronidazole loaded Span 60 organogel. ${ }^{23}$ Pseudo-plastic flow behavior was observed in sesame oil based Span 60-Carbopol bigels, a desirable characteristic of semisolid formulations, due to the rearrangement of dispersed droplets resulting in the breakdown of bigger droplets into the smaller ones after application of higher shear force without loss of structural integrity. ${ }^{8}$ It was also observed that viscosity increased with increase in Span 40/Tween 80 proportion in oleogels of olive oil. Uvanesh et al. reported that the increase in Span 60 concentration in oleogels resulted in improved firmness and mechanical strength due to increase in the junction points amongst the fibrous structures. ${ }^{24}$ Addition of Tween 80 is responsible for the formation of flexible mesh-like self-assembled structure within gel network in olive oil. Hybrids exhibited 2-5 times greater viscosity than their corresponding oleogels indicating better mechanical strength. Increase in viscosity was observed with higher percentage of HPMCK15M in olive gel hybrids. Modeling of viscosity data revealed nonNewtonian pseudo-plastic flow behavior $(n<1)$.

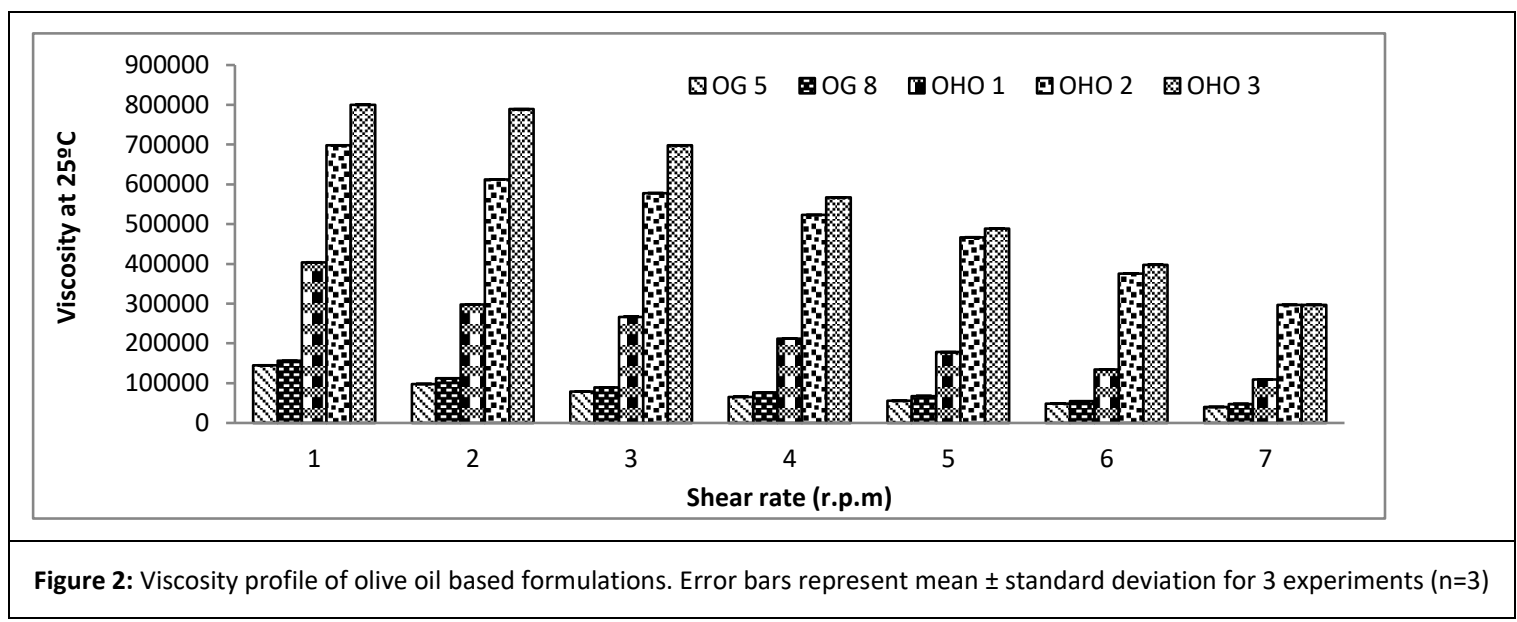

\section{Thermal analysis}

The gelation kinetics was studied by monitoring the change in turbidity during transformation of the blank gel to its sol state on cooling. For OG5, the gelation time was $1440 \mathrm{~s} \pm 1.6$ which was higher than that of OG8 (1160s \pm 1.8$)$ containing higher $\%$ of gelator-cogelator in the ratio of 2:1. Olive oil parameter $(\alpha)$ and organogelator-co-gelator parameter $(\beta)$ values obtained from Gompertz model is presented in Table 2.Gel-sol transition temperature $\left(\mathrm{T}_{\mathrm{g}}\right)$ value was found to increase with increase in gelator/cogelator concentration as observed from Table 2. Melt flow index of olive oleogels was found to decrease with increase in gelator/co-gelator concentration. The order of melt flow index is described as follows:OG $5(4.8 \mathrm{~g} / 10 \mathrm{~s}$ $\pm 0.3)>$ OG $8(3.5 \mathrm{~g} / 10 \mathrm{~s} \pm 0.6)$

DSC profiles of Span, oleogel and oleohydrogel hybrid are represented in Figure 3. The thermal analysis parameters (onset temperature, melting temperature and melting enthalpy) of OG 5 and OHO 1 are tabulated in Table 2 . The hybrid formulation demonstrated initiation of melting at lower temperature and very low value of heat of enthalpy in comparison to oleogel indicating lower thermal stability and probably more flexible micro architecture. Thermal analysis of olive oil based oleogels 
suggested that increase in Span 40/Tween 80 concentration decreased gelation time and caused gel to sol transformation to occur at higher temperature indicating that self-assembly in the gel state is governed by strong intermolecular interactions attributable to Span 40 or inter-aggregate interactions in case of Tween $80 .{ }^{25}$ The transformation process is reversible as the gels reformed on cooling exhibiting same properties as before. As the concentration of gelator/co-gelator was increased, $\alpha$-value decreased with lower melt flow index indicating formation of comparatively compact and rigid gel network with higher viscosity and better thermal stability as manifested in increasing $\beta$-value and higher $T_{g}$ (Table
2). The heating DSC curves of the olive oil based Span/ Tween oleogels showed a sharp endothermic peak in the temperature range of $47-55 \circ \mathrm{C}$ (Figure 3 ) proving the improved thermal stability as manifested by high $\beta$-value. The melting range of olive gel hybrid (OHO 1 ) was found to be $46 \stackrel{\circ}{\circ}$. DSC study revealed that the change in the enthalpies with hybrid $(\Delta \mathrm{Hm})$ was found to be quite low in comparison to the native oleogel of olive oil because less energy was necessary for crystal disorganization of hybrids containing HPMC with high water retaining capacity. ${ }^{26}$ Therefore, hybrids may be assumed to be less rigid or more flexible compared to Span/Tween containing oleogel with expected better drug release.

Table 2: Thermal analysis of olive oil based formulations

\begin{tabular}{|c|c|c|c|c|c|c|}
\hline \multirow{3}{*}{$\begin{array}{l}\text { Batch } \\
\text { OG } 5\end{array}$} & \multirow{3}{*}{$\begin{array}{l}T_{g}(\circ C) \\
50 \pm 0.3\end{array}$} & \multicolumn{2}{|c|}{ Modeling of kinetics } & \multirow{3}{*}{$\begin{array}{c}T_{\text {onset }}(\stackrel{\circ}{ } \mathbf{C}) \\
48\end{array}$} & \multirow{3}{*}{$\begin{array}{l}\mathrm{T}_{\mathrm{m}}(\stackrel{\circ}{ } \mathbf{C}) \\
55.91\end{array}$} & \multirow{3}{*}{$\begin{array}{r}\Delta H_{m}(\mathrm{~J} / \mathrm{g}) \\
46.1253\end{array}$} \\
\hline & & $\alpha$ & $\beta$ & & & \\
\hline & & 2.5702 & 0.1801 & & & \\
\hline OG 8 & $56 \pm 0.3$ & 2.2104 & 0.2043 & - & - & - \\
\hline $\mathrm{OHO} 1$ & - & - & - & 39 & 46.42 & 9.6345 \\
\hline $\mathrm{OHO} 2$ & - & - & - & - & - & - \\
\hline $\mathrm{OHO} 3$ & - & - & - & - & - & - \\
\hline
\end{tabular}

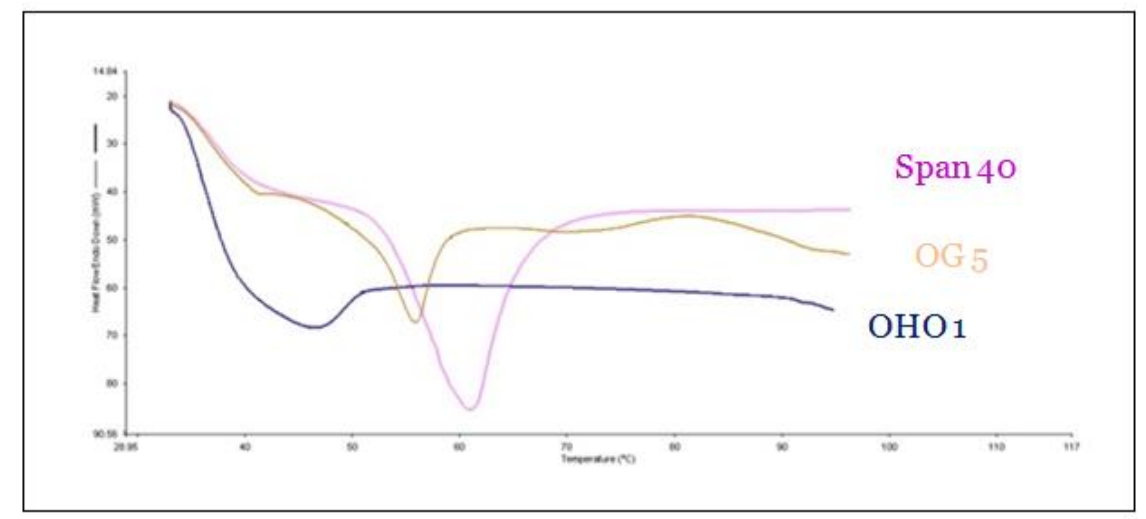

\section{Diffusion study}

The drug (PCM) release profile from oleogels and oleohydrogel hybrids is graphically presented in Figure 4. Increase in gelator- co-gelator concentration lowered the drug release. Higher concentration of HPMCK15M in oleohydrogel hybrid retarded drug release. Kinetic modeling of the drug release data from the formulations is given in Table 3.Drug diffusion parameters of olive oil based formulations are tabulated in Table 3. Release of PCM from oleogels and oleohydrogel hybrids depends upon the solubility and partition coefficient of the PCM in olive oil. ${ }^{27}$ In vitro release study revealed that concentration-independent drug release from oleogels decreased with increase in Span 40/Tween 80 concentrations. For olive oleogels, maximum drug release was achieved from $\mathrm{OG}^{*} 5$ with $\mathrm{t}_{50}$ of 3.4 hand no lag time could be detected in release profile. This indicates immediate drug permeation across synthetic membrane attributed to beneficial effect of oleic acid component of olive oil in oleogel. Olive oil is reported to exhibit superior physical and chemical stability and improved permeation across the skin for hydroquinone topical creams compared to semisolid preparations containing Beeler's base. ${ }^{28}$ It can also be suggested that Span and Tween induced alteration of barrier properties of the stratum corneum, change in partition coefficient of vehiclestratum corneum and also better skin hydration. However, higher concentration of gelator-cogelator resulted in formation of a rigid gel with impaired rug release profile. ${ }^{29}$ Modeling of gelation kinetics data revealed lower $\alpha$ - value with increasing concentration of gelator suggesting formation of more rigid and compact gel-network that retarded drug release from the topical vehicles. Similar observations were reported by Satapathy et al in their study on release of metronidazole from mustard oil and groundnut oil based Span 40 organogels. ${ }^{14}$ As OG*5 showed satisfactory drug release profiles amongst all oleogels of olive oil, it was selected for formulation of oleohydrogel hybrids by employing 3 different $\% \mathrm{w} / \mathrm{v}$ of HPMCK15M hydrogels. The oleohydrogel hybrid $\left(\mathrm{OHO}^{*} 1\right)$ exhibited improved $\mathrm{PCM}$ 
release $(87 \%)$ and higher values of $\mathrm{SS}_{\text {flux }}$ and $\mathrm{K}_{\mathrm{p}}$ compared to their native oleogel containing Span-Tween (in the ratio of 2:1). This can be attributed to several factors such as flexible microarchitecture of the hybrid, solvent imbibition facilitated by hydrophilic HPMC and further emulsification promoted by the surfactant-type gelator/co-gelator molecules, which might have happened earlier at the hybrid interface between the oleogel and hydrogel enabling faster and better drug release than from oleogel. Hybrid formulations with higher percentage of hydrophilic swellable HPMC K15M ( 3 and $4.5 \% \mathrm{w} / \mathrm{v}$ ) formed matrix diffusion barrier and demonstrated lower drug release than the corresponding oleogel with Span-Tween. Only the hybrids with $1.5 \% \mathrm{w} / \mathrm{v}$ HPMC showed superior drug release following zero-order kinetics with non-Fickian diffusion. Singh et al. reported that metronidazole (2 gm) loaded sesame oil-Span 60 oleogel $(2.02 \mathrm{gm})$ and Carbopol hydrogel (17.78 gm) based bigel showed $100 \pm 0.02 \%$ drug release at the end of $1.5 \mathrm{hr}$ following Higuchi kinetics with non-Fickian diffusion. ${ }^{8}$

Table 3: Drug release kinetics and drug diffusion parameters of gels

\begin{tabular}{|c|c|c|c|c|c|}
\hline Batch & $\begin{array}{l}\text { Kinetics } \\
\text { followed }\end{array}$ & $\begin{array}{l}\text { Diffusion } \\
\text { mechanism }\end{array}$ & $t_{50} *(h)$ & $\begin{array}{c}\text { SS }_{\text {flux }}{ }^{*} \\
\left(\mathrm{mg} / \mathrm{cm}^{2} / \mathrm{h}\right)\end{array}$ & $\mathrm{K}_{\mathrm{p}} *\left(\mathrm{~cm}^{2} / \mathrm{h}\right)$ \\
\hline$O G * 5$ & Zero & Fickian & $3.4 \pm 0.2$ & $3.52 \pm 0.5$ & $1.76 \pm 0.4$ \\
\hline OG* 8 & Zero & Non-Fickian & $3.9 \pm 0.3$ & $3.29 \pm 0.2$ & $1.64 \pm 0.5$ \\
\hline OHO* 1 & Zero & Non-Fickian & $3.6 \pm 0.2$ & $3.94 \pm 0.3$ & $1.97 \pm 0.3$ \\
\hline OHO* 2 & Higuchi & Non-Fickian & $4.2 \pm 0.6$ & $2.67 \pm 0.2$ & $1.33 \pm 0.6$ \\
\hline OHO* 3 & Higuchi & Non-Fickian & $5.4 \pm 0.7$ & $2.59 \pm 0.8$ & $1.29 \pm 0.2$ \\
\hline
\end{tabular}

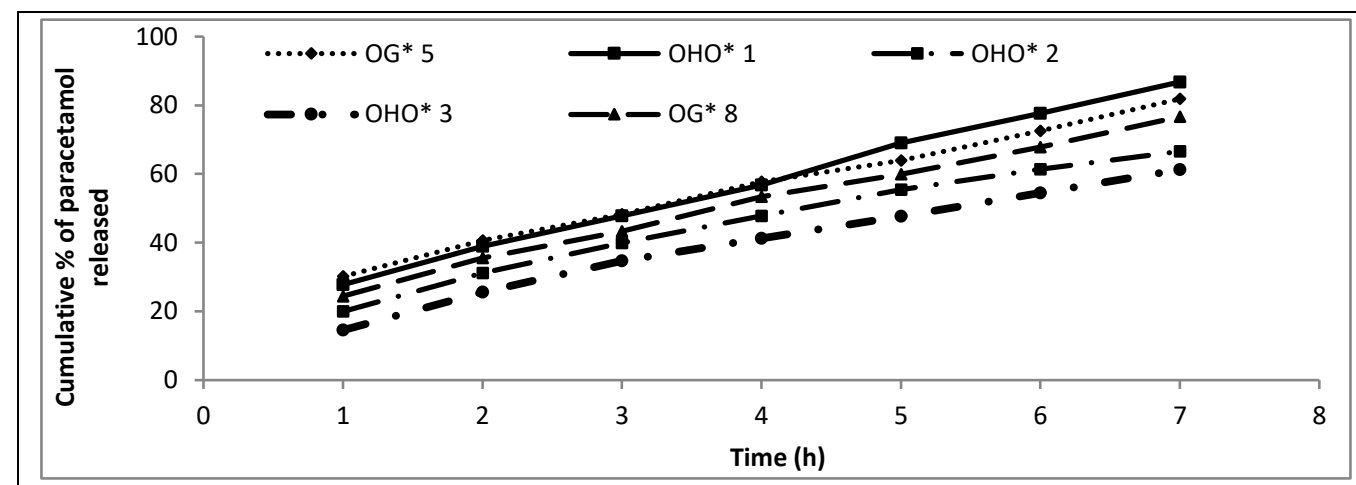

Figure 4: Drug release profiles of olive oil based formulations in phosphate buffer $(\mathrm{pH}$ 5.8). Error bars represent mean \pm standard deviation for 3 experiments $(n=3)$

\section{Accelerated stability study}

Olive oil based oleogels and oleohydrogel hybrid were found to be stable up to 6 cycles upon exposure to freezethaw cycles (Figure 5).The hypothesis of the thermocycling stability study (freeze-thaw) is that the gelator / gelator-co-gelator layer, which is primarily a surfactant, may get damaged due to the change in the physicochemical properties thereby leading to the instability of the formulations when subjected to frequent temperature changes. ${ }^{13}$ This study helps to predict the mechanism of destabilization of the formulations due to the change in the physicochemical properties at the extreme conditions. The chances of oxidative changes are predominant at higher temperatures whereas the formation of solidified structures at lower temperatures may alter the physical interactions amongst the sample components responsible for the formation of the networked structures. ${ }^{14}$ From accelerated thermal stability study, it was observed that olive oil based oleogels were found to be stable up to 7 cycles (here 6 cycles reported graphically) (Figure 5) although there was a significant increase (10-45\%) in gelation time from 4 th cycle onwards. This might be attributed to too much rigidity in the gel microstructure which once disrupted during thawing took more time to build up or reform. Satapathy et al. stated that mustard oil and ground nut oil based Span 40 organogels were stable for more than 8 cycles. ${ }^{14}$ However, in hybrid formulations; phase separation was detected after 6 cycles which is obvious due to immiscibility of apolar oil/oleogel phase and aqueous gel/sol phase. Singh et al. reported that all the sesame oil based metronidazole loaded stearic acid/ Carbopol bigels retained their structural integrity after completion of five thermo-cycles, suggesting good thermal stability. ${ }^{8}$ The $\%$ change of gelation time of hybrids was found to be increased by 2.5-3.3 times than corresponding oleogels. The $\Delta \mathrm{H}_{\mathrm{m}}$ value of $\mathrm{OHO} 1$ was found to be less compared to their corresponding oleogels indicating lower thermal stability. This justifies the fact that the accelerated stability study on hybrids 
could not be carried out for more than 6 cycles after which phase separation was observed. Thus, in order to ensure better shelf-life stability of oleohydrogel hybrids, temperature fluctuations should be avoided and preferably be stored at room temperature.

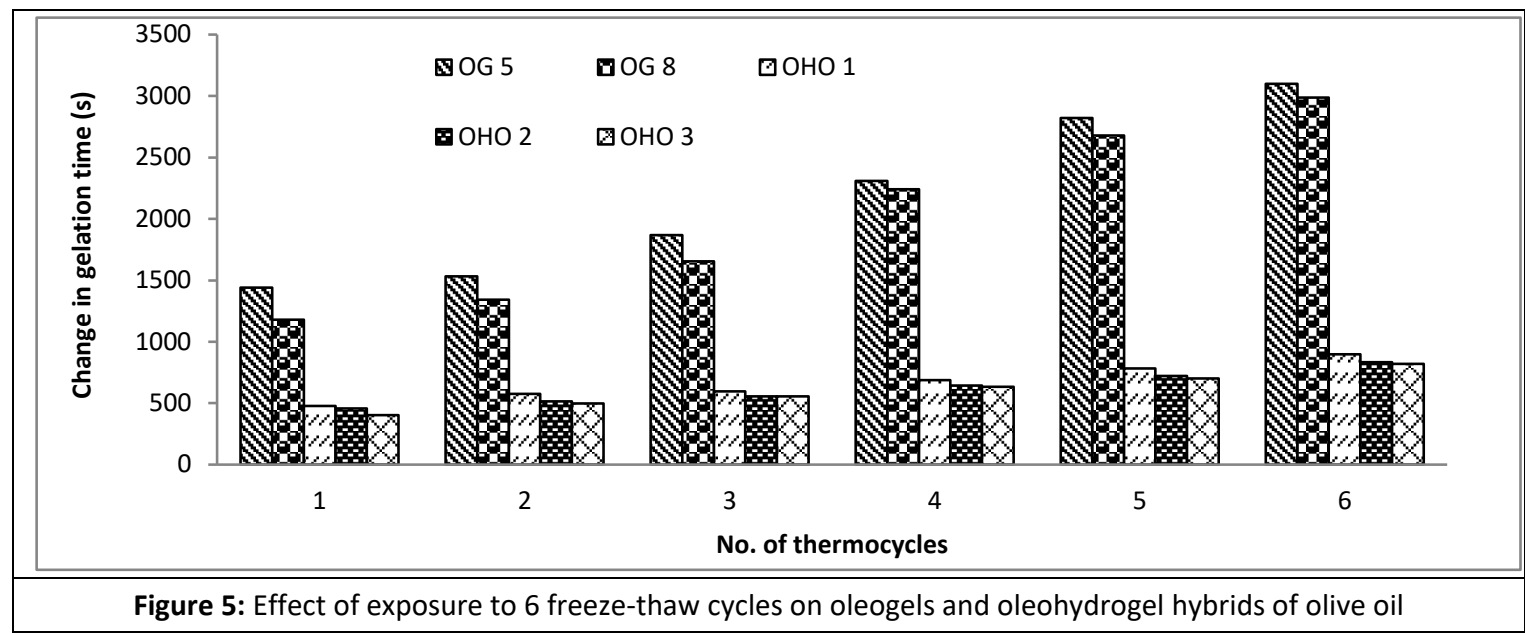

Thus, olive oil based oleohydrogel hybrid constituted a novel topical base with flexible microarchitecture as corroborated by simultaneous observations for thermal analysis, rheological studies and drug release profile. Moreover, presence of specific fatty acid in olive oil, surfactant type organogelator-cogelator molecules in the novel base might alter the physical state and permeability of the skin via hydration and modification of lipid bilayer structure of stratum corneum. ${ }^{30}$

\section{CONCLUSION}

From the various studies, it can be concluded that incorporation of hydrogel component with HPMCK15M as the gelling polymer to form olive oleohydrogel hybrids has altered the microarchitecture of the native oleogel and produced significant improvement in rheological behaviour as well as drug diffusion parameters. Therefore, oleohydrogel hybrids may be regarded as novel topical base that may be employed to improve drug release from topical formulations.

\section{REFERENCES}

1. Jang $\mathrm{HH}$, Lee $\mathrm{SN}$, Epidermal skin barrier, Asian Journal of Beauty and Cosmetology, 14, 2016, 339-47.

2. Machado ACHR, Lopes PS, Raffier CP, Haridass IN, Roberts M, Grice J, Silva L, Skin penetration, In: Sakamoto K, Lochhead R, Maibach H, Yamashita Y,(eds.). Cosmetic Science and technology: theoretical principles and application, US: Elsevier, 2017, 741-55.

3. Rehman $\mathrm{K}$ and Zulfakar $\mathrm{MH}$, Recent advances in gel technologies for topical and transdermal drug delivery, Drug Development and Industrial Pharmacy, 40, 2014, 433-40.

4. Bajerski L, Michels LR, Colome LM, Bender EA, Freddo RJ, Bruxel $F$, The use of Brazilian vegetable oils in nanoemulsion: an update on preparation and biological applications,
Brazilian Journal of Pharmaceutical Sciences, 52(3), N2016,348-63.

5. Behera B, Dey S, Sharma V, Pal K, Rheological and viscoelastic properties of novel sunflower oil-Span 40biopolymer-based bigels and their role as a functional material in the delivery of antimicrobial agents, Advances in Polymer Technology, 34, 2015, 1-10.

6. Behera B, Singh VK, Kulanthaivel S, Bhattacharya MK, Paramanik K, Banerjee I, Pal k, Physical and mechanical properties of sunflower oil and synthetic polymers based bigels for the delivery of nitroimidazole antibiotic-a therapeutic approach for controlled drug delivery, European Polymer Journal, 64, 2015, 253-64.

7. Ibrahim MM, Hafez SA, Mahdy MM, Organogels, hydrogels and bigels as transdermal delivery systems for diltiazem hydrochloride, Asian Journal of Pharmaceutical Sciences, 8 , 2013, 48-57.

8. Singh VK, Anis A, Banerjee I, Paramanik K, Bhattacharya MK, Pal K, Preparation and characterization of novel Carbopol based bigels for topical delivery of metronidazole for the treatment of bacterial vaginosis, Material Sciences andEngineering C, 44, 2014, 151-8.

9. Diaz-Valenzuela A, Garcia-Fernandez FP, CarmonaFernandez PJ, Vlle-Canete MJ, Pancorbo-Hidalgo PL, Effectiveness and safety of olive oil preparation for topical use in pressure ulcer prevention: multicentre, controlled, randomized and double- blinded clinical trial, International Wound Journal, 16(7), 2019, 1-9.

10. Smaoni S, Hima HB, Jarraya R, Kamoun NG, Ellouze R, Damak $M$, Cosmetic emulsion from virgin olive oil: formulation and bio-physical evaluation, African Journal of Biotechnology, 11(34), 2012, 8417-24.

11. Pawar KR, JayachandraBabu R, Lipid materials for topical and transdermal delivery of nanoemulsion, Critical 
Reviews $^{\mathrm{TM}}$ in Therapeutic Drug Carrier System, 31(5), 2014, 429-54.

12. Singh VK, Behera B, Sagiri SS, Pal K, Anis A, Bhattacharya MK, Organogels in controlled drug delivery, In: Mishra $M$ (ed). Handbook of encapsulation and controlled release, London, UK. CRC Press, 2015, 1035-66.

13. Sah SK, Badola A, Mukhopadhyay S, Development and evaluation of tioconazole loaded emulgel, International Journal of Applied Pharmaceutics, 9, 2017, 83-90.

14. Satapathy D, Sagiri SS, Pal K, Paramanik K,Development of mustard oil and groundnut oil-based Span 40 organogels as matrices for controlled drug delivery, Designed Monomers and Polymers, 17, 2014, 545-56.

15. Balata GF and Shamardl HAEM,Formulation of cholorpheniramine maleate in Span 60/ Tween 20 based organogels for transdermal delivery, Journal of Innovations in Pharmaceutical and Biological Sciences, 4, 2017, 49-57.

16. Indian Pharmacopoeia. Government of India, Ministry of Health and Family Welfare Department, 7th edition, 1, 2014, 559-621.

17. Shukla AK, Yadav SK, Tiwari V,Linear models for S-shaped growth curves, Journal of Statistics Applications and Probability, 4, 2015, 113-7.

18. Shah DK, Sagiri SS, Behera B, Pal K, Pramanik K, Development of olive oil based organogels using sorbitan monopalmitate and sorbitan monostearate: a comparative study, Journal of Applied Polymer Science, 129, 2013, 793804.

19. Singh R, Kumar R, Friction welding of dissimilar plastic-based material by metal powder reinforcement, In: Hashmi MSJ (ed). Reference module in materials science and materials engineering,Dublin, Ireland. Elsevier, 2017, 1-16.

20. Bartosova L, Bajgar J, Transdermal drug delivery in vitro diffusion cells, Current Medicinal Chemistry, 19, 2012, 46717.

21. Kopanichuk LV, Vedenchuk EA, Koneva AS, Vanin AA, Structural properties of Span 80/ Tween 80 reverse micelles by molecular dynamics simulations, The Journal of Physical Chemistry B, 122, 2018, 8047-55.
22. Phadtare D, Phadtare G, Nilesh B, Asawat M, Hypromellosea choice of polymer in extended release tablet formulation, World Journal of Pharmacy and Pharmaceutical Sciences, 3, 2014, 551-66.

23. Singh VK, Pramanik K, Ray SS, Pal K, Development and characterization of sorbitan monostearate and sesame oilbased organogels for topical delivery of antimicrobials, AAPS PharmSciTech, 16, 2014, 293-305.

24. Uvanesh K, Sagiri SS, Senthilguru K, Pramanik K, Banerjee I, Anis A, Zahrani S, Pal K, Effect of Span 60 on the microstructure, crystallization kinetics, and mechanical properties of stearic acid oleogels: an in-depth analysis, Journal of Food Science, 81, 2016, 380-7.

25. Trivedi DR, Ballabh A, Dastidar P, Ganguly B, Structureproperty correlation of a new family of organogelators based on organic salts and their selective gelation of oil from oil/water mixtures, Chemistry-A European Journal, 10, 2004, 5311-22.

26. Cruz AA, Montealvo GM, Feria JS, Perez LAB, Effect of carboxy methyl cellulose and xanthan gum on the thermal, functional and rheological properties of dried nixtamalised maize masa, Carbohydrate Polymer, 62, 2005, 222-31.

27. Bhattacharya C, Kumar N, Sagiri SS, Pal K, Ray SS, Development of Span 80-Tween 80 based fluid-filled organogels as a matrix for drug delivery, Journal of Pharmacy and Bioallied Science, 4, 2012, 155-63.

28. Serrano DR, JoseGordo M, Matji A, Gonzalez S, Lalatsa A, Torrado JJ, Tuning the transdermal delivery of hydroquinone upon formulation with novel permeation enhancers, Pharmaceutics, 11, 2019, 1-16.

29. Akram MR, Ahmad M, Abrar A, Sarfraz RM, Mahmood A, Formulation design and development of matrix diffusion controlled transdermal delivery of glimepiride, Drug design, development and Therapy, 12, 2018, 349-64.

30. Zsiko S, Csauyi E, Kovacs A, Szucs MB, Gacsi A, Berko S, Methods to evaluate skin permeation in vitro, Scientia Pharmaceutica,87, 2019, 1-21.

Source of Support: None declared.

Conflict of Interest: None declared.

For any question relates to this article, please reach us at: editor@globalresearchonline.net

New manuscripts for publication can be submitted at: submit@globalresearchonline.net and submit_ijpsrr@rediffmail.com 